\title{
Téoros
}

Revue de recherche en tourisme

\section{Grande vitesse ferroviaire et tourisme, des relations conditionnées et à double sens Le cas d'Anaheim (Californie)}

\section{Charlotte Ruggeri}

Volume 32, numéro 2, 2013

Tourisme et transport

URI : https://id.erudit.org/iderudit/1036594ar

DOI : https://doi.org/10.7202/1036594ar

Aller au sommaire du numéro

Éditeur(s)

Université du Québec à Montréal

ISSN

0712-8657 (imprimé)

1923-2705 (numérique)

Découvrir la revue

Citer cet article

Ruggeri, C. (2013). Grande vitesse ferroviaire et tourisme, des relations conditionnées et à double sens : le cas d'Anaheim (Californie). Téoros, 32(2), 47-57. https://doi.org/10.7202/1036594ar
Résumé de l'article

Si les acteurs locaux ont souvent tendance à tout attendre en termes de développement économique de la grande vitesse ferroviaire, le cas d'Anaheim (Californie) démontre que le tourisme peut aussi servir d'argument pour défendre un projet de ligne et de gare à grande vitesse. Le tourisme et les loisirs deviennent alors une opportunité pour stimuler la construction d'infrastructures de transport, ou pour repenser les réseaux existants. Cet article se propose donc d'étudier les rapports entre grande vitesse ferroviaire et tourisme, sous l'angle des politiques d'aménagement d'infrastructures entrepris par les acteurs locaux dans le cadre du projet de ligne à grande vitesse en Californie. Cette étude a été réalisée au cours d’une thèse de doctorat en géographie et à partir d'un travail de terrain, essentiellement centré sur des entretiens menés en 2012 et 2013. Ce projet permet de mettre en évidence les conditions d'élaboration d'une relation grande vitesse ferroviaire-tourisme dans le cadre d'un contexte touristique favorable. Toutefois, l'exemple d'Anaheim démontre également que la grande vitesse ferroviaire renforce les activités touristiques préexistantes plus qu'elle ne les initie. Enfin, cette étude insiste sur la nécessité de penser des infrastructures de transport intégrées aux territoires et réseaux locaux afin d'optimiser les bénéfices attendus sur le tourisme.
Ce document est protégé par la loi sur le droit d'auteur. L’utilisation des services d’Érudit (y compris la reproduction) est assujettie à sa politique d'utilisation que vous pouvez consulter en ligne.

https://apropos.erudit.org/fr/usagers/politique-dutilisation/ 


\title{
Grande vitesse ferroviaire et tourisme, des relations conditionnées et à double sens Le cas d'Anaheim (Californie)
}

\author{
Charlotte RUGGERI \\ Doctorante en géographie \\ Laboratoire MRTE - Mobilités, Réseaux, Territoires, Environnement \\ Université de Cergy-Pontoise (France) \\ charlotte.ruggeri@gmail.com
}

\begin{abstract}
RÉSUMÉ: Si les acteurs locaux ont souvent tendance à tout attendre en termes de développement économique de la grande vitesse ferroviaire, le cas d'Anaheim (Californie) démontre que le tourisme peut aussi servir d'argument pour défendre un projet de ligne et de gare à grande vitesse. Le tourisme et les loisirs deviennent alors une opportunité pour stimuler la construction d'infrastructures de transport, ou pour repenser les réseaux existants.

Cet article se propose donc d'étudier les rapports entre grande vitesse ferroviaire et tourisme, sous l'angle des politiques d'aménagement d'infrastructures entrepris par les acteurs locaux dans le cadre du projet de ligne à grande vitesse en Californie. Cette étude a été réalisée au cours d'une thèse de doctorat en géographie et à partir d'un travail de terrain, essentiellement centré sur des entretiens menés en 2012 et 2013. Ce projet permet de mettre en évidence les conditions d'élaboration d'une relation grande vitesse ferroviaire-tourisme dans le cadre d'un contexte touristique favorable. Toutefois, l'exemple d'Anaheim démontre également que la grande vitesse ferroviaire renforce les activités touristiques préexistantes plus qu'elle ne les initie. Enfin, cette étude insiste sur la nécessité de penser des infrastructures de transport intégrées aux territoires et réseaux locaux afin d'optimiser les bénéfices attendus sur le tourisme.
\end{abstract}

Mots-clés: acteur, localité, Anaheim, Disneyland, grande vitesse, train, transport.

\section{Introduction et problématisation}

La grande vitesse ferroviaire peut être couramment associée à un miracle économique pour les territoires qu'elle dessert. En effet, lorsque des projets de lignes ferroviaires à grande vitesse et de gares sont développés sur des territoires, les acteurs locaux espèrent que la grande vitesse sera synonyme de développement économique. Les avantages de la grande vitesse ferroviaire sont nombreux et favorisent l'accessibilité de certains territoires. Les trains à grande vitesse permettent d'atteindre plus rapidement les villes desservies. Ils permettent souvent d'arriver en plein centre des villes, contrairement au transport aérien. Si l'on voyage seul ou à deux, le train à grande vitesse revient moins cher que l'automobile. Ces trois principaux arguments sont souvent pertinents, mais ne s'appliquent pas systématiquement à tous les territoires desservis. En effet, les gares exurbanisées sont courantes sur les lignes à grande vitesse. Par ailleurs, le train à grande vitesse n'est pas toujours la solution la plus économique selon le pays concerné. Au-delà de ces arguments qui relèvent de la pratique, un autre élément joue en faveur de la grande vitesse ferroviaire : les réseaux sont rentables dans les pays où ils sont implantés, particulièrement en France (CHSRA, 2012). Au vu de ces arguments, on peut comprendre l'intérêt et le désir des territoires d'être reliés aux réseaux de la grande vitesse ferroviaire.

De fait, tous ces arguments prennent également sens pour le tourisme. Puisque le tourisme implique un déplacement de son lieu d'habitation vers un lieu de villégiature ou de loisirs, les transports sont au cour de cette activité. Ainsi, la recherche en géographie s'est penchée sur les liens entre transports et tourisme (Équipe MIT, 2002; Duhamel et Knafou, 2007), en particulier entre grande vitesse ferroviaire et tourisme (Chen et Haynes, 2012; Coronado et al., 2012; Delaplace et Perrin, 2012). Ces recherches ont surtout été menées à partir de cas européens puisqu'en Europe, le réseau à grande vitesse existe depuis le début des années 1980. Cet intérêt à étudier la relation entre ce mode de transport et le tourisme reflète les espoirs que les acteurs locaux formulent envers la grande vitesse ferroviaire. Certains acteurs locaux associent la grande 
vitesse à un «miracle» économique et touristique puisque ces trains permettent - selon eux - de diversifier les types de séjours et la clientèle, d'augmenter le nombre de touristes, mais aussi de mettre en valeur de nouveaux territoires du tourisme (Dunn, 2012; Lai, 2012 : communications personnelles). Mais qu'en est-il réellement? Quels sont les possibles impacts de la grande vitesse ferroviaire sur l'activité touristique d'une ville desservie et comment les villes tentent-elles de s'emparer de ces opportunités de développement touristique?

Cet article se propose d'étudier les rapports entre grande vitesse ferroviaire et tourisme sous l'angle des politiques d'aménagement d'infrastructures entrepris par les acteurs locaux. En effet, la recherche en géographie montre que le lien entre tourisme et grande vitesse ferroviaire n'est pas évident et relève de relations conditionnées par des stratégies locales importantes. Pour ce faire, cet article entend traiter du cas d'Anaheim, ville touristique de Californie qui a décidé de se saisir de l'opportunité que représente le futur train à grande vitesse dans cet État.

\section{Méthodologie}

Les données de cette étude sont issues d'un travail de recherche mené entre 2011 et 2013 sur le terrain, en Californie, et d'une littérature grise importante. Mener une étude sur un projet institutionnel implique tout d'abord de partir des documents sources publiés par les responsables politiques du projet : l'État californien et le gouvernement fédéral (CHSRA, 2011, 2012 et 2014; U.S. Department of Transportation, 2009, 2010 et 2013). Ces rapports se doublent de nombreux rapports universitaires ou issus d'agences de conseil qui cherchent à mettre en évidence les conséquences et les impacts du projet de grande vitesse ferroviaire, que ce soient des conséquences positives ou négatives (Orange Business Council, 2008; Catz et Christian, 2010). Le projet étant récent, peu d'ouvrages ou d'articles scientifiques traitent de la grande vitesse aux ÉtatsUnis ou en Californie. Les écrits d'Anthony Perl permettent tout de même d'avoir une vision historique assez complète de l'évolution des politiques publiques ferroviaires (Perl, 2002 et 2012). Enfin, la presse quotidienne fournit un bon baromètre de l'évolution de la perception du projet en relatant les avancées ou reculades du projet, ainsi qu'en se faisant le porteparole des acteurs locaux et régionaux.

De fait, les acteurs locaux et régionaux constituent la source principale qui permet de cerner les enjeux territoriaux du projet sur le terrain. Ces acteurs dépendent d'échelles variées : l'échelle fédérale, l'échelle californienne, l'échelle des Metropolitan Planning Organizations (MPO - ce sont des agences fédérales regroupant plusieurs comtés et chargées de l'aménagement des transports) (Caltrans, 2013), l'échelle des comtés et l'échelle des villes. Les acteurs étudiés sont soit responsables du projet ou liés à l'autorité, ou encore concernés par les impacts du projet, c'est-à-dire d'un territoire que le train devrait traverser ou desservir. À l'issue de deux séjours en Californie en 2012 et 2013, j'ai pu rencontrer une cinquantaine d'acteurs interrogés lors d'entretiens semi-directifs. Toutefois, dans cet article, je m'en tiendrai à la dizaine d'acteurs concernés par le projet d'Anaheim, en y ajoutant certains acteurs du corridor Los Angeles-San Diego.
Le terrain de recherche s'est centré sur deux espaces : la Vallée centrale où doit commencer la construction du train à grande vitesse et qui est le territoire le plus concerné par l'opposition au projet actuellement; la Californie du Sud, déjà choisie comme corridor ferroviaire de la grande vitesse dans les années 1980 et territoire le plus peuplé de l'État et où des projets annexes de grande vitesse voient le jour (vers Las Vegas notamment). J'ai centré mes recherches plus particulièrement sur le corridor Los Angeles-Anaheim et Los Angeles-San Diego où les acteurs se sont montrés dynamiques et impliqués dans le processus.

En effet, sur ce corridor, si l'autorité a identifié douze villes comme gares potentielles (CHSRA, 2014), les acteurs s'accordent pour dire que seules deux ou trois villes devraient obtenir une gare entre Los Angeles et San Diego. De fait, toutes ces villes sont dans une politique de lobby auprès de l'autorité pour être desservies par le train à grande vitesse à partir des années 2020-2030. Anaheim fait partie de ces villes en campagne.

Pour cerner ce terrain sud-californien, plusieurs entretiens ont été menés sur place et permettent de comprendre l'intérêt touristique et économique d'Anaheim d'être intégrée au projet de grande vitesse. Tout d'abord, des entretiens ont été réalisés à Anaheim avec la municipalité (Lai, 2012 : communications personnelles). J'ai rencontré, entre autres, des responsables de l'aménagement du territoire et des travaux de l'ARTIC (Alpine et Davis, 2013 : communications personnelles); des consultants qui travaillent pour l'autorité sur la section Los Angeles-Anaheim (Labrado, 2013 : communications personnelles); des acteurs locaux du comté d'Orange et de la MPO de la Southern California Association of Governments (SCAG) (Dunn, 2012 et Fox, 2012 : communications personnelles); des acteurs locaux des municipalités du corridor sud (Backstrom, 2013; Gardner, 2013; Gutierrez, 2013; Montanez, 2013; Petrek, 2013 et Wapner, 2013 : communications personnelles), et enfin des journalistes (Vartabedian, 2012 : communications personnelles). Pourtant, le lien entre grande vitesse ferroviaire et dynamisme touristique n'est pas avéré, encore moins systématique.

\section{La grande vitesse ferroviaire et le tourisme : une relation pas toujours évidente}

La recherche en géographie s'intéresse depuis plusieurs années aux liens et aux potentiels impacts de la grande vitesse ferroviaire sur le développement économique, en particulier sur le tourisme (Chen et Haynes, 2012; Coronado et al., 2012; Delaplace et Perrin, 2012). Ces recherches sont avant tout menées à partir de cas européens puisque ce sont des espaces où la grande vitesse est développée depuis une trentaine d'années (Chen et Haynes, 2012).

\section{La grande vitesse ferroviaire : une infrastructure de transport pour quel type de tourisme?}

Si le lien entre tourisme et grande vitesse relève donc avant tout d'un cycle positif, les recherches menées en Europe démontrent que seuls trois types de tourisme peuvent véritablement bénéficier de la grande vitesse. Ainsi, le tourisme d'affaires et de congrès, le tourisme de court séjour et le tourisme à la 
journée sont potentiellement sensibles à l'arrivée de la grande vitesse sur un territoire (Coronado et al., 2012). Le tourisme d'affaires concerne les personnes qui sont en déplacement professionnel. La grande vitesse, avec ses temps de transport réduits, offre alors un avantage certain puisqu'elle permet de réaliser plus d'allers et retours dans la journée (Duhamel et Knafou, 2007). Le tourisme de courte durée joue également sur cet avantage, mais la concurrence avec l'automobile existe, notamment en termes de coûts (Coronado et al., 2012). À plus de deux personnes, l'automobile se montre plus rentable, mais la grande vitesse ferroviaire reste concurrentielle puisqu'elle évite les encombrements routiers et les problèmes de stationnement (Delaplace et Perrin, 2012). Enfin, le tourisme à la journée est généralement celui qui profite le plus de la grande vitesse ferroviaire. Dans tous les cas, ce tourisme «à grande vitesse» induit souvent une réduction des nuitées sur place (Coronado et al., 2012).

Malgré cela, les différentes études montrent que la relation entre tourisme et grande vitesse ferroviaire n'est pas automatique. Ce mythe des effets structurants des infrastructures de transports sur les activités touristiques est remis en cause ou questionné (Offner, 1993; Bazin et al., 2011; anonyme, 2014). Certes, de nouvelles dynamiques peuvent émerger, mais elles sont conditionnées (Delaplace et Perrin, 2012).

\section{Territoires, tourismes et grande vitesse : des relations conditionnées}

La recherche sur la grande vitesse ferroviaire affirme que celle-ci peut avoir des impacts sur le développement de l'activité et de la fréquentation touristique, mais cela relève souvent de trois éléments : des potentialités locales importantes, des stratégies locales en amont de l'arrivée de la grande vitesse et le ciblage des clientèles les plus concernées (Masson et Petiot, 2009).

Il faut tout d'abord que le territoire desservi offre des aménités touristiques de valeur (Coronado et al., 2012). Dans le même ordre d'idée, la ville doit disposer d'une capacité hôtelière importante en termes de quantité, mais aussi de qualité, afin de plaire à tous les touristes potentiels (Delaplace et Perrin, 2012).

Le deuxième élément permettant une relation positive entre grande vitesse ferroviaire et tourisme est l'implication en amont des acteurs locaux (Bazin et al., 2011). Les acteurs locaux, généralement les élus municipaux, doivent prévoir l'arrivée de la grande vitesse ferroviaire et penser des politiques publiques et privées permettant le développement économique local (Bazin et al., 2010). Une nouvelle desserte ferroviaire représente des potentialités pour les territoires locaux, mais la concrétisation de ces opportunités dépend de la volonté et de l'action des acteurs (Bazin et al., 2010). La réussite des territoires locaux à se saisir des opportunités que représente la grande vitesse ferroviaire dépend en grande partie des stratégies locales mises en œuvre (Urena et Ribalaygua, 2004).

Ces stratégies locales relèvent de l'appropriation du projet par les acteurs locaux. Cette appropriation peut revêtir plusieurs formes : une appropriation individuelle relevant d'un acteur privé ou public ou une appropriation collective permettant une coordination de plusieurs acteurs autour d'un même projet. Une action isolée est rarement pertinente et les acteurs locaux doivent prendre conscience de la nécessité de construire un projet d'accompagnement de la nouvelle desserte ferroviaire cohérent à l'échelle du territoire (Bazin et al., 2010).

Enfin, la dernière stratégie qui permet de bénéficier de la grande vitesse est le ciblage de la clientèle. En effet, la grande vitesse concerne avant tout trois types de tourisme : le tourisme d'affaires, le tourisme urbain et le tourisme à la journée (Coronado et al., 2012). De fait, les acteurs locaux, publics ou privés, doivent cibler ces clientèles (Masson et Petiot, 2009). Cela peut consister en des réductions sur les hôtels lorsque l'on réserve son billet de train ou des réductions sur le billet d'entrée dans les attractions de loisirs (Coronado et al., 2012). Ce ciblage passe également par des campagnes de promotion de la destination, qui peuvent être menées par l'opérateur du train lui-même, par les acteurs locaux comme la municipalité ou des acteurs privés (Delaplace et Perrin, 2012). La participation des acteurs privés peut aussi passer par l'organisation d'événements, notamment d'événements nocturnes, comme les parades, qui permettent de retenir les touristes pour la nuit (Coronado et al., 2012). Enfin, à terme, les acteurs locaux comme la municipalité ou les acteurs privés doivent faciliter le séjour des touristes en organisant, par exemple, un accueil des touristes en gare (Delaplace et Perrin, 2012).

\section{La gare : I'enjeu central de la relation grande vitesse ferroviaire-tourisme}

La question de la gare est donc au centre des relations entre grande vitesse ferroviaire et tourisme, puisque c'est par cette infrastructure que les touristes qui choissisent le train comme mode de transport arrivent. Construire une gare à grande vitesse, que ce soit en vue de soutenir une activité touristique ou non, pose deux enjeux primordiaux pour les territoires urbains : l'intégration territoriale des gares aux réseaux de transports locaux, et aux dynamiques urbaines locales (Facchinetti-Mannone et Richer, 2011). En effet, pour que la relation entre tourisme et grande vitesse soit bénéfique, la gare à grande vitesse ne doit pas être pensée comme un îlot, mais plutôt comme une porte d'entrée sur les activités de loisirs et de tourisme de la ville.

Ces éléments sont d'autant plus vrais pour le tourisme à la journée. Le temps gagné par la rapidité de la grande vitesse ne doit pas être gâché par l'attente en gare ou par des transports en commun peu performants sur place (L'Hostis et Baptiste, 2006). De fait, la localisation d'une gare à grande vitesse relève d'enjeux d'accessibilité et d'organisation territoriale primordiaux. Les acteurs locaux doivent penser cette nouvelle infrastructure comme un moyen d'optimiser les potentialités et les réseaux locaux (Facchinetti-Mannone et Richer, 2011). De fait, les acteurs locaux ne doivent pas négliger la question de l'accessibilité à la gare. Cet élément est très important puisqu'il permet à la gare de s'intégrer au territoire qu'elle dessert. Une intermodalité performante est nécessaire dans les gares à grande vitesse parce qu'elles polarisent un espace qui dépasse l'échelle locale (FacchinettiMannone et Richer, 2011). 


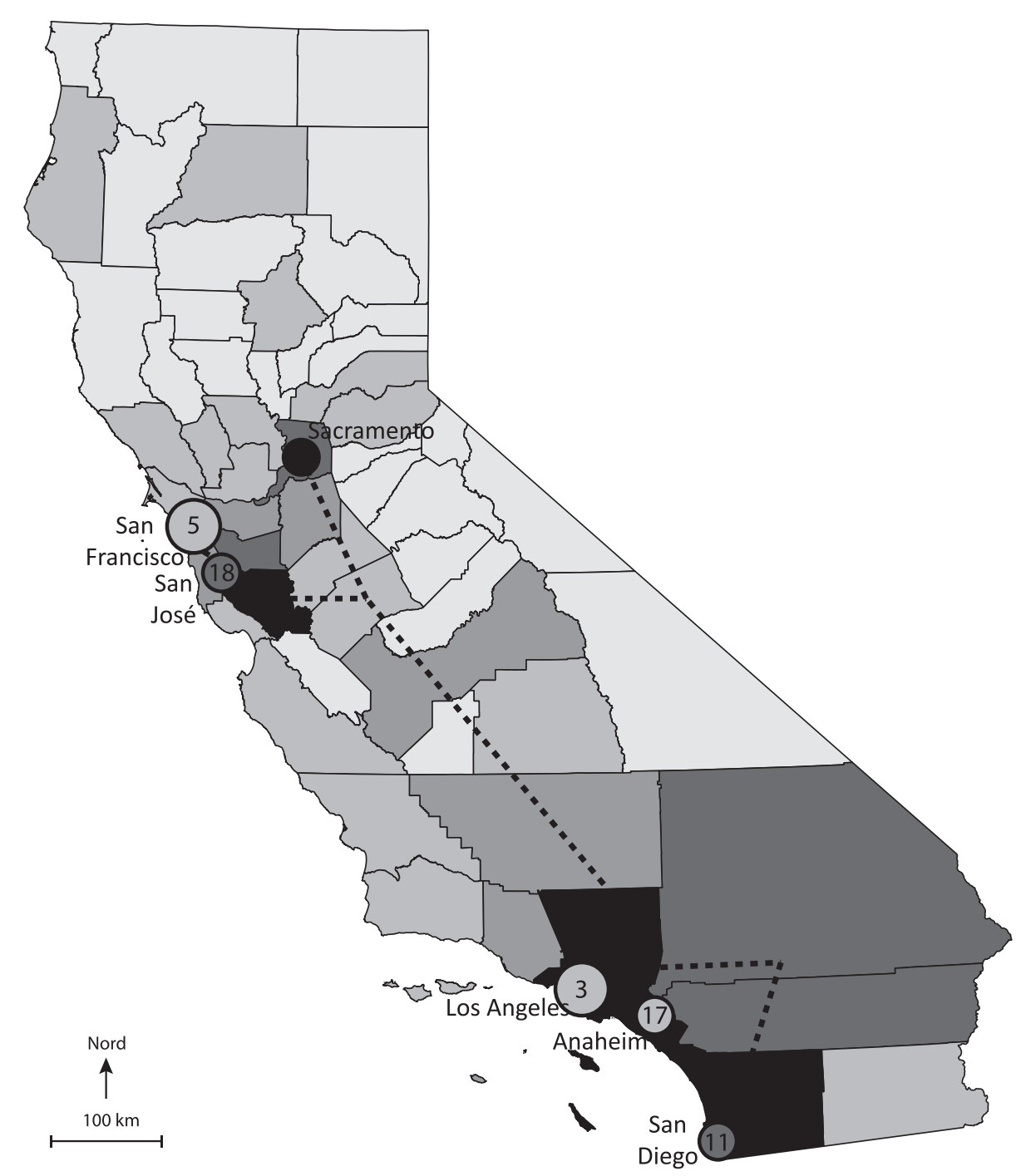

Recettes touristiques par comté en 2012

Plus de 2 milliards de dollars de recettes

Entre 1 et 2 milliards de dollars de recettes

Entre 500 millions et 1 milliard de dollars de recettes

Entre 100 et 500 millions de dollars de recettes

Moins de 100 millions de dollars de recettes

Le projet de grande vitesse ferroviaire

Tracé proposé par l'Autorité californienne

de la grande vitesse ferroviaire

Fréquentation touristique par ville en 2012

1 million de touristes

500000 touristes

Évolution du nombre de touristes positive entre 2000 et 2012

Évolution du nombre de touristes négative entre 2000 et 2012

3) Classement parmi les 20 premières villes touristiques des États-Unis

ILLUSTRATION 1 : La Californie, un État touristique (source : Ruggeri (2014)).
La relation entre grande vitesse ferroviaire et tourisme peut donc faire émerger des dynamiques urbaines, économiques et de transports, selon certaines conditions. Si ces conditions existent, on peut alors se demander quels seront les effets sur la destination touristique (Anaheim), mais aussi pour le train à grande vitesse.

\section{Anaheim : un pôle touristique états-unien et californien}

La ville d'Anaheim en Californie, accueille le parc de Disneyland depuis 1955. Elle a été choisie comme cas d'étude pour cet article. Anaheim n'est cependant pas un cas isolé en Californie, puisque le Golden State est le troisième État touristique en termes de fréquentation (U.S. Department of Commerce, 2012). 
Tableau 1: La fréquentation des parcs à thème aux États-Unis

\begin{tabular}{c|c|c|c}
\hline Rang & Parc & Ville et État & Nombre de visiteurs en millions \\
\hline 1 & Magic Kingdom (DisneyWorld) & Lake Buena Vista, Floride & 18,5 \\
\hline 2 & Disneyland & Ananeim, Californie & 16,2 \\
\hline 3 & EPCOT (DisneyWorld) & Lake Buena Vista, Floride & 11,2 \\
\hline 4 & Disney's Animal Kingdom & Lake Buena Vista, Floride & 10,1 \\
\hline 5 & Disney's Hollywood Studios & Ananeim, Californie & 8,1 \\
\hline 6 & Disney's California Adventure & Orlando, Floride & 8,5 \\
\hline 7 & Islands of Adventure (Universal) & Orlando, Floride & 7 \\
\hline 8 & Universal Studios & Universal City, Californie & 6,1 \\
\hline 9 & Universal Studios Hollywood & Orlando, Floride & 5 \\
\hline 10 & SeaWorld & San Diego, Californie & 4,3 \\
\hline 11 & SeaWorld & & \\
\hline
\end{tabular}

Source : Ruggeri (2014), d'après la Themed Entertainment Association (2014).

\section{Le tourisme californien : une activité variée et puissante}

La Californie dispose de nombreux atouts touristiques : des plages; des parcs nationaux nombreux tels que Yosemite, la Vallée de la Mort, King's et Sequoia, Joshua Tree; des villes au patrimoine important comme San Francisco; ainsi que des parcs d'attraction, très répandus dans le sud de l'État. La Californie offre donc des paysages et des activités touristiques très diversifiés qui peuvent attirer de nombreux touristes (Foucrier et Coppolani, 2004; Foucrier, 2010).

Historiquement, le train a permis de rendre les lieux du tourisme californiens plus accessibles. En effet, les compagnies ferroviaires états-uniennes et californiennes ont, dès la fin du XIX siècle, promu le train comme le moyen privilégié de l'accès aux parcs nationaux et aux plages californiens (Starr, 1985; Foucrier, 2010). À partir des années 1950, les compagnies aériennes jouent sur les mêmes procédés publicitaires, mettant en scène les paysages grandioses des parcs et des plages sur leurs affiches.

De fait, le tourisme est une activité majeure de l'économie californienne depuis la fin du XIX ${ }^{e}$ siècle. En 2012, la California Travel \& Tourism Commission estime que le tourisme représente 32,3 milliards de dollars de recettes dans l'État et 917000 emplois (CTTC, 2013). S'il est difficile d'obtenir le chiffre du nombre de touristes visitant la Californie par an, on peut se reférer à d'autres chiffres pour prendre conscience de l'importance du secteur. En termes de transports, la Californie a enregistré 31 millions de personnes arrivées par avion sur le territoire pour y effectuer un séjour touristique en 2012 (CTTC, 2013). Il est aussi intéressant de prendre en compte les données du secteur hôtelier, puisqu'en 2012, la demande en chambre oscille entre 28 millions pour le trimestre hivernal (janvier, février et mars) et 35 millions pour le trimestre estival (juillet, août et septembre) (CTTC,
2013). Cette variation assez faible entre les deux saisons s'explique par le climat relativement stable et chaud toute l'année dans une grande partie de la Californie (CTTC, 2013). Dans ce contexte très touristique de la Californie, plusieurs territoires se distinguent, notamment le comté d'Orange et Anaheim.

\section{Orange et Anaheim, berceaux \\ du tourisme récréatif états-unien}

Le comté d'Orange se hisse en effet à la seconde place californienne en termes de recettes touristiques en 2012, derrière le comté de Los Angeles et devant celui de San Diego (CTTC, 2013). En termes d'emplois, le comté totalise 83000 emplois dans le secteur touristique, ce qui la classe quatrième dans l'État, après San Francisco (183 000 emplois), Los Angeles (160 000) et San Diego (117 000) (CTTC, 2013). La Californie se distingue également par ces villes, dont cinq font partie des vingt villes les plus touristiques des États-Unis : Los Angeles, San Francisco, San Diego, Anaheim et San José (U.S. Department of Commerce, 2012) (Illustration 1).

Anaheim se hisse dans ce classement grâce à plusieurs attractions touristiques dont les parcs à thème. Le tourisme à Anaheim repose en effet sur une forme récréative particulière, celle du parc à thème et des loisirs sportifs. Rémy Knafou (2003) définit le parc à thème comme "un espace clos d'accès payant destiné au tourisme et au loisir et proposant des activités récréatives». La Californie du Sud regorge de parcs à thème comme les parcs Universal, SeaWorld ou encore Six Flags. Les quatre principaux parcs du sud de la Californie ont attiré 35,1 millions de personnes en 2013 et se classent parmi les vingt-cinq parcs les plus fréquentés au monde (Themed Entertainment Association, 2014). Ils représentent presque un tiers de la fréquentation des parcs à thème des États-Unis, comme le montre le tableau 1. 


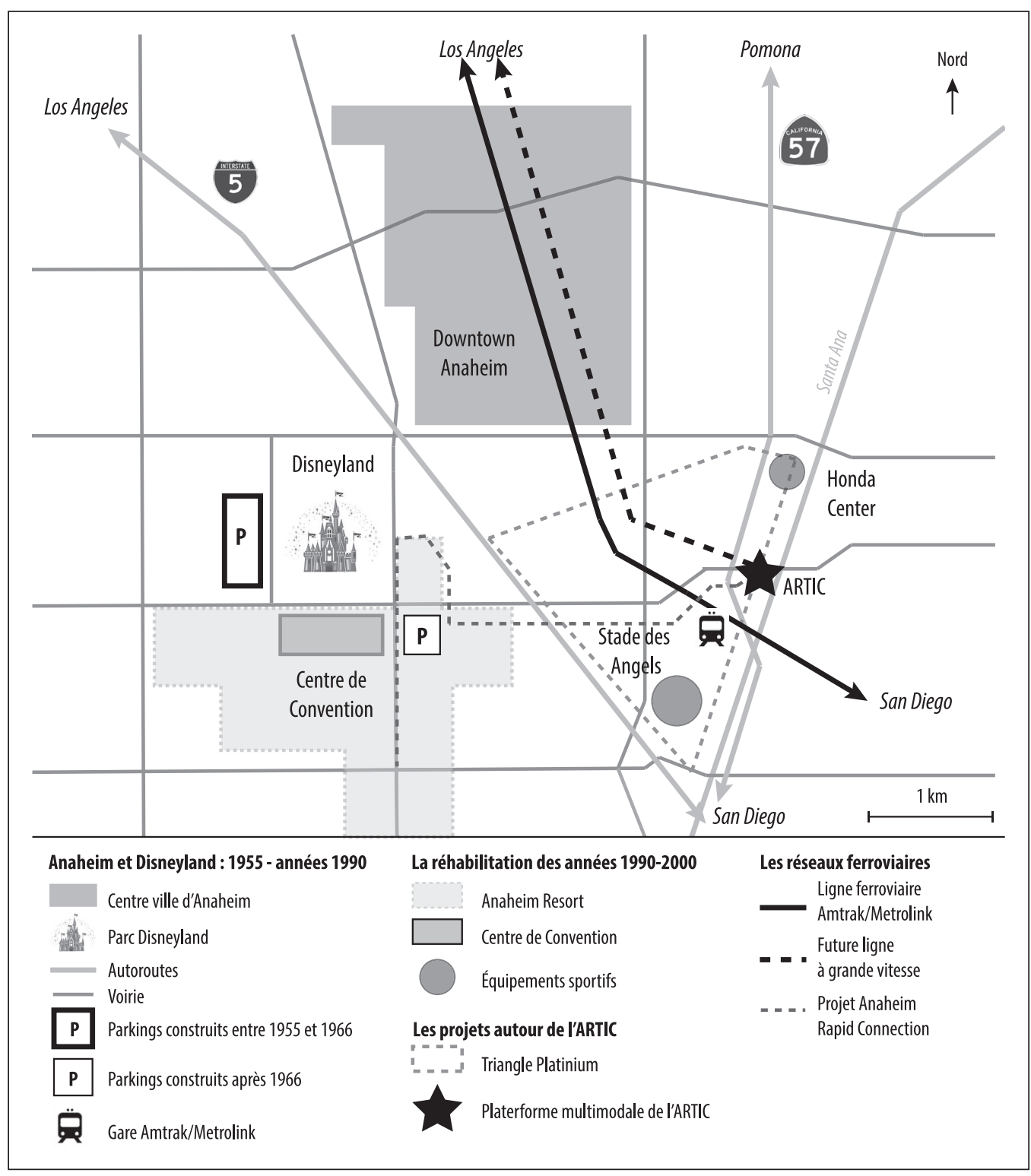

ILLUSTRATION 2 : Anaheim et Disney : acteurs de la requalification urbaine? (source : Ruggeri (2014)).
À Anaheim, ce tourisme récréatif se double d'équipements sportifs de loisirs qui attirent à leur tour une population nombreuse : le stade des Angels, l'équipe de baseball, et le Centre Honda, ouvert en 1993, qui accueille l'équipe de hockey des Ducks (voir Illustration 2). Certes, ces activités de loisirs attirent une population nombreuse les jours de match, mais elles n'ont pas le même impact sur l'économie touristique de la ville, puisque cette population recourt faiblement aux hébergements. Enfin, Anaheim s'est affirmée dans les années 1990 comme une ville touristique d'affaires avec le Centre de convention, ouvert en 1967, et agrandi successivement en 1993 et 1997.

Afin de répondre à cette fréquentation touristique, les hébergements sont présents en quantité puisque le site internet Expedia recense 94 hôtels à Anaheim (recherche effectuée en ligne en décembre 2013). Si l'on s'intéresse de près à cette hôtellerie, on remarque une concentration des établissements autour des parcs de Disneyland, sur les avenues Katella et Harbor notamment (voir Illustration 3). Ces hôtels sont des deux étoiles et la nuit coûte 78 euros en moyenne. La distance moyenne entre les parcs Disneyland et les hôtels est de 1,4 kilomètres.

En termes de transport, la ville est actuellement desservie par des infrastructures routières et ferroviaires importantes. Elle est connectée à l'autoroute majeure de la Californie, l'Interstate 5, qui la relie à Los Angeles et au nord de la Californie, ainsi qu'à San Diego, dans le sud. Les transports ferroviaires ne sont pas absents du comté d'Orange et de la ville puisque des trains Amtrak et Metrolink, le réseau régional de Los Angeles, arrivent à quelques kilomètres du parc de Disneyland. Actuellement, les mobilités urbaines sont avant tout assurées par un réseau d'autobus. Anaheim est également à quelques 
ILLUSTRATION 3 : Une hôtellerie au service de Disneyland? (source : Ruggeri (2014)).

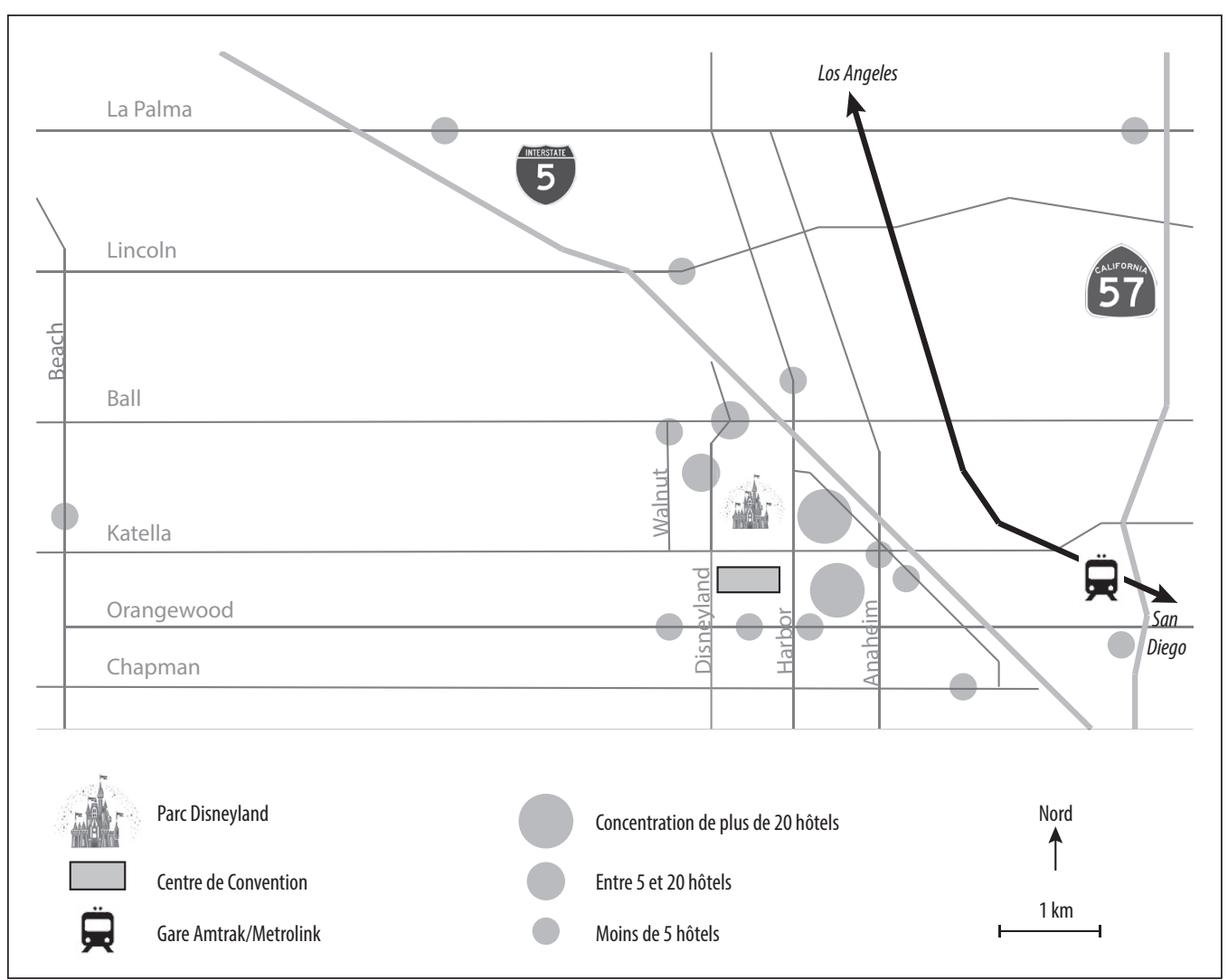

kilomètres du sixième aéroport californien, l'aéroport John Wayne de Santa Ana (California Airports Council, 2011). Si la ville semble déjà bien desservie par plusieurs modes et infrastructures de transports, on peut se demander pourquoi la ville d'Anaheim mène depuis 2008 une campagne intense pour accueillir une gare du futur train à grande vitesse californien.

\section{Le cas d'Anaheim ou une relation duale : renforcer les pôles touristiques par la grande vitesse / justifier la grande vitesse par le tourisme}

L'arrivée de la grande vitesse entraîne généralement un renforcement des activités et de la fréquentation touristiques des villes (Chen et Haynes, 2012). Les villes sans intérêt touristique ne deviennent pas magiquement dignes d'intérêt pour les touristes lorsqu'elles sont desservies par un train à grande vitesse (Coronado et al., 2012). De fait, on peut penser que la relation entre la grande vitesse ferroviaire et Anaheim est double ou duale, elle bénéficie à la fois aux activités touristiques de la ville et au projet de grande vitesse en Californie.

\section{Anaheim, une ville mobilisée}

\section{pour la grande vitesse ferroviaire}

La ville d'Anaheim a commencé sa campagne de lobby en 2008 en présentant son projet ARTIC (Anaheim Regional Transportation Intermodal Center), alors même que le projet de grande vitesse semblait incertain. Sa campagne en faveur du train s'avère plutôt réussie puisque lorsque l'Autorité pensait supprimer la section Los Angeles-Anaheim (CHSRA, 2011), les acteurs locaux sont parvenus à faire réintégrer cette section dans le projet de grande vitesse (CHSRA, 2012). Ainsi, parmi toutes les villes du corridor sud, Anaheim a sa propre section, sorte d'appendice à la ligne à grande vitesse, et est presque assurée de voir des trains à grande vitesse la desservir dans les années 2020. Anaheim serait ainsi accessible en 25 minutes à partir de la gare d'Union Station de Los Angeles. À l'instar des villes entre Los Angeles et San Diego, qui devront se mobiliser dans la décennie à venir pour s'imposer sur le tracé, Anaheim s'est mobilisée très tôt en présentant un projet de gare ambitieux. L'argument des acteurs locaux pour réintégrer le tracé et justifier la construction de la gare était simple: Anaheim et le comté d'Orange estiment recevoir entre 40 et 45 millions de touristes par an (ARTIC, 2012; Anaheim Orange County Visitor \& Convention Bureau, 2013).

Dans le cas d'Anaheim, les enjeux touristiques ont favorisé l'implantation et le maintien de la ligne à grande vitesse au détriment d'autres espaces (Delaplace et Perrin, 2012) : "Le seul motif, c'est Disneyland, l'économie et le tourisme» (Fox, 2012 : communications personnelles). Par cette phrase, Stephen G. Fox résume ce que de nombreux observateurs et acteurs locaux du sud de la Californie pensent de la section Los Angeles-Anaheim. De fait, certains acteurs du corridor sud veulent s'inspirer de la campagne d'Anaheim pour garder sa gare comme les élus de Riverside (Gardner, 2013; Gutierrez, 2013 : communications personnelles). Les villes plus «assurées» de voir le train à grande vitesse s'arrêter sur leur territoire, comme Ontario, qui dispose d'un aéroport, pensent 


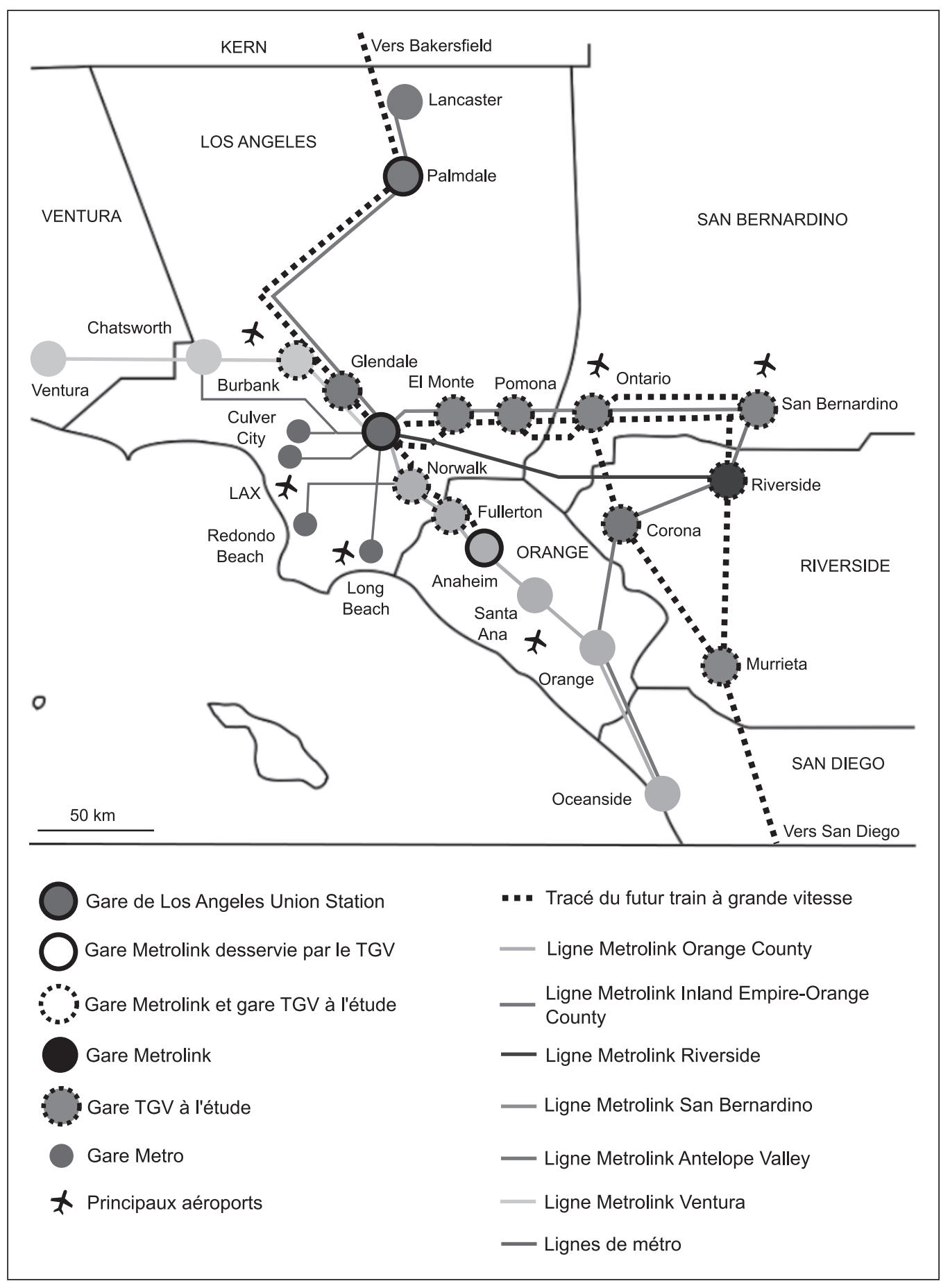

ILLUSTRATION 4 : Les réseaux de transport du sud californien (source : Ruggeri (2013)). d'ailleurs qu'au-delà de la grande vitesse, l'enjeu est de renforcer leurs connexions avec Anaheim par le biais du Metrolink (voir illustration 4) ou du projet de Super Speed Train vers Las Vegas (Montanez, 2013 et Wapner, 2013 : communications personnelles).

Le train à grande vitesse n'est donc pas perçu comme un moyen de générer une activité touristique à Anaheim, mais comme un moyen de soutenir une croissance économique forte : «Le comtéd'Orange est très peuplé (...) et a une croissance économique très forte. Abandonner le comté d'Orange n'a pas plus de sens que de maintenir une gare à Fresno" (Vartabedian, 2012 : communications personnelles). Les transports ne sont pas considérés comme la clé de l'activité économique, plutôt comme le moyen d'intensifier et de renouveler la fréquentation touristique en Californie du Sud, mais aussi d'offrir une alternative de transports aux touristes (Équipe MIT, 2002). 
Le nouveau train pourrait permettre à plus de personnes de se rendre dans les divers équipements récréatifs de la ville : "Anaheim accueille 40 millions de visiteurs par an et la moitié va à Disneyland. Le train à grande vitesse permettrait de déposer les touristes à la porte du parc Disney, donc oui, le premier argument est touristique. Il s'agit avant tout de faciliter les transports pour les touristes» (Lai, 2012 : communications personnelles). Le train permettrait ainsi d'échapper à la congestion des autoroutes donnant accès à la ville, saturées depuis 1955 et l'inauguration du parc de Disneyland (Marling, 1991; Didier, 2002). Selon Lai (2012), "Le problème de la congestion est insoluble, donc il faut réfléchir à un nouveau mode de transport». L'ARTIC est donc avant tout conçu comme «une alternative de transport permettant d'accéder aux événements et destinations de l'Anaheim Resort, du stade des Angels et du Centre Honda» (OCTA, 2012).

Toutefois, la grande vitesse et la gare prévues à cet effet ne font pas tout. Arrivé à destination, le touriste doit pouvoir utiliser les transports en commun urbains pour accéder aux lieux touristiques (Coronado et al., 2012). Une fois la gare à grande vitesse obtenue, l'enjeu pour Anaheim est de penser à des liaisons locales et régionales pertinentes, mais aussi de penser à l'environnement urbain autour de la gare (FacchinettiMannone et Richer, 2011).

\section{Tourisme, grande vitesse ferroviaire et intégration locale}

Les concepteurs de l'ARTIC n'ont pas délaissé l'enjeu de l'intégration locale. La municipalité voudrait se poser en tête de pont du changement des pratiques de mobilité : "Le projet de l'ARTIC fera d'Anaheim et du comté d'Orange des modèles en termes d'organisation des transports» (OCTA, 2012). Dans un espace dont la croissance économique et l'urbanisation ont été initiées et ont reposé sur l'extension du réseau routier, c'est un tournant radical (Le Goix, 2003). En termes de transports, l'ARTIC est une infrastructure novatrice présentée comme " ambitieuse» et "progressiste» par les acteurs locaux (Dunn, 2012; Lai, 2012; Alpine et Davis, 2013 et Labrado, 2013 : communications personnelles).

Avec une construction lancée en septembre 2012 par la ville et l'OCTA (Orange County Transportation Authority), l'ARTIC, centre régional intermodal, permettrait de connecter en une plateforme plusieurs réseaux de transports. Tout d'abord, elle accueillerait les deux réseaux ferroviaires : le Metrolink, qui dessert la Californie du Sud ainsi que l'Amtrak, qui dessert la Californie et le reste du pays. L'ARTIC disposerait également de plusieurs plateformes pour les bus de l'OCTA qui desservent le comté et ceux d'Anaheim. Le trafic Metrolink/Amtrak est déjà dense, puisqu'en 2013, Amtrak comptabilisait 342284 embarquements à Anaheim (Amtrak, 2013). À terme, la plateforme devrait également recevoir le train à grande vitesse californien, mais aussi l'ARC (Anaheim Rapid Connection) et le California-Nevada Super Speed Train. L'ARC est un tramway qui desservirait l'Anaheim Resort et le Triangle Platinium, les deux espaces réhabilités ou en cours de réhabilitation d'Anaheim (voir Illustration 2). Sa construction devrait commencer en 2014 et la ligne de 3,5 miles (5,6 kilomètres) devrait entrer en service en 2018 (OCTA, 2012).
En Californie, peu de villes peuvent aujourd'hui présenter un projet de plateforme intermodale complète et moderne comme l'ARTIC, qui devrait accueillir cinq réseaux de transports différents.

\section{Un projet de requalification urbaine à long terme}

Le site choisi pour l'ARTIC entre dans un projet plus large visant à repenser le quartier des parcs Disneyland (voir Illustration 2). Cette politique de requalification a été amorcée dans les années 1990.

Si la ville considèrait dès 1953 l'installation du parc comme une aubaine touristique, elle n'a pas saisi cette opportunité pour redynamiser plus largement l'économie de la ville et du quartier. C'est seulement une quarantaine d'années plus tard que la municipalité lance un premier projet de réhabilitation urbaine avec l'Anaheim Resort (Didier, 2000). Cette politique fait suite à la décision de la compagnie Disney d'ouvrir un second parc, le Disney's California Adventure, et un Downtown Disney, espace de commerces, restaurants et loisirs à l'entrée gratuite. Anaheim pense alors que les espaces entourant le parc doivent eux aussi faire peau neuve et profiter des millions de visiteurs des parcs. La municipalité s'associe donc avec la compagnie Disney dans le cadre d'un partenariat public-privé pour aménager des hôtels, restaurants et rues commerçantes autour du parc (Didier, 2001).

Ce quartier, en réhabilitation depuis vingt ans, est logiquement choisi pour accueillir l'ARTIC, mais le projet doit entraîner la requalification d'un espace adjacent, le Triangle Platinium (voir Illustration 2). Cet espace est, pour ainsi dire, presque vide actuellement. Il n'accueille que deux grandes infrastructures, le stade des Angels et le Centre Honda, qui attirent du monde les jours de match. L'enjeu de l'intégration urbaine de la gare se situe donc dans cet espace, à relier et à connecter à l'Anaheim Resort et au centre d'Anaheim. Ce territoire pourrait ainsi devenir un lieu complémentaire intéressant pour l'activité touristique de la ville en polarisant tous les réseaux de transport, mais aussi un moyen de diversifier l'activité locale. En effet, les concepteurs de l'ARTIC pensent qu'au delà des activités touristiques, le site pourrait attirer des entreprises et leurs bureaux (Alpine et Davis, 2013 : communications personnelles). Ainsi, la chambre de commerce du comté d'Orange estime que près de 20000 emplois pourraient voir le jour grâce à l'arrivée de la grande vitesse (Orange County Business Council, 2008; Catz et Christian, 2010). Toutefois, comme pour le tourisme, les impacts économiques de la grande vitesse, notamment sur la création d'emplois, sont conditionnés et ne relèvent pas du miracle économique (Chen et Hall, 2012).

\section{Conclusion}

Le cas d'Anaheim démontre bien que la grande vitesse ferroviaire ne produit pas le tourisme. Dans des territoires fortement irrigués par des réseaux de transports, l'ajout d'une nouvelle infrastructure de transport permet avant tout d'intensifier l'activité touristique et de la diversifier. Anaheim semble avoir compris que l'enjeu touristique de la grande vitesse reposait avant tout sur sa capacité à se saisir de cette opportunité. De fait, la ville présente aujourd'hui le projet 
de gare le plus ambitieux (avec San Francisco), mais surtout le plus avancé. Elle est devenue un modèle à suivre pour les autres villes intermédiaires du tracé qui veulent démontrer leur capacité à accueillir le futur train à grande vitesse (Ontario, Riverside, San Bernardino, Corona, Murrieta). Avec le projet - actuellement très embryonnaire - de Super Speed Train vers Las Vegas, un corridor touristique de la grande vitesse pourrait même voir le jour et faire d'Anaheim le pôle intermodal du tourisme en Californie du Sud.

Mais au-delà de ces considérations locales plutôt positives, le cas d'Anaheim démontre que le lien entre tourisme et grande vitesse entraîne avant tout un renforcement des pôles touristiques préexistants. De plus, même si le train à grande vitesse ne voit jamais le jour en Californie, Anaheim restera un pôle touristique indéniable, ce qui relativise quelque peu l'enjeu de la grande vitesse sur ce territoire et fournit un bon argument aux opposants au train. Le train à grande vitesse est donc surtout un accélérateur du tourisme : il permet d'accéder plus vite à la destination, de multiplier les séjours sur place, de diversifier les types de séjours, de repenser les transports locaux et d'offrir des opportunités économiques dans des quartiers en réhabilitation. De fait, la relation est à double sens : la grande vitesse profite au tourisme et le tourisme profite à la grande vitesse. Mais de manière assez paradoxale, la grande vitesse et ses infrastructures pourraient aussi permettre à ce territoire monofonctionnel de diversifier ses activités économiques.

\section{Références}

AMTRAK (2013) Amtrak Fact Sheet, Fiscal Year 2013, State of California, Washington D.C.: Amtrak Government Affairs. 6 p.

ANAHEIM ORANGE COUNTY VISITOR \& CONVENTION BUREAU (2013) 2013 Economic Impact of Tourism in Orange County, Orange: Anaheim Orange County Visitor \& Convention Bureau. 1 p.

Anonyme (2014) «Les effets structurants des infrastructures de transport », L'espace géographique, tome 43, no. 1. p. 51-67.

ARTIC (2012) ARTIC Project Development Benefits. Anaheim: AConnext. $3 \mathrm{p}$.

BAZIN, Sylvie; Christophe BECKERICH et Marie DELAPLACE (2010)

«Desserte ferroviaire à grande vitesse, activation des ressources spécifiques et développement du tourisme : le cas de l'agglomération rémoise », Belgéo, n¹-2, p. 65-77.

BAZIN, Sylvie; Corinne BLANQUART; Christophe BECKERICH; Marie DELAPLACE et Ligdwine VANDENBOSSCHE (2011) «Grande vitesse ferroviaire et développement économique local : une revue de littérature», Recherche Transports Sécurité, vol. 27, n³, p. 215-238.

CAC - CALIFORNIA AIRPORTS COUNCIL (2011) Annual Report 2011, Sacramento: California Airports Council. 7 p.

CHSRA - CALIFORNIA HIGH-SPEED RAIL AUTHORITY (2011) California High-Speed Rail Program Draft 2012 Business Plan, Sacramento: California High-Speed Rail Authority. 230 p.

CHSRA - CALIFORNIA HIGH-SPEED RAIL AUTHORITY (2012) California High-Speed Rail Program Revised 2012 Business Plan, Building California's Future, Sacramento: California High-Speed Rail Authority. 212 p.

CHSRA - CALIFORNIA HIGH-SPEED RAIL AUTHORITY (2014) Connecting California, Draft 2014 Business Plan, February 7, Sacramento: California High-Speed Rail Authority. 101 p.
CTTC - CALIFORNIA TRAVEL \& TOURISM COMMISSION (2013) California Travel Impacts by County, 1992-2011, 2012 Preliminary State \& Regional Estimates, Sacramento: California Travel \& Tourism Commission/California Business, Transportation and Housing Agency, Division of Tourism. $170 \mathrm{p}$.

CALTRANS - California Department of Transportation, Division of Transportation Planning (2013) California Metropolitan Planning Organizations (MPOs) and Regional Transportation Planning Agencies (RTPAs), Sacramento: California Department of Transportation. 1 p.

CATZ, Sarah L. et Adam CHRISTIAN (2010) Thinking Ahead, HighSpeed Rail in Southern California, Irvine: UC Irvine, Institute of Transportation Studies. 28 p.

CHEN, Chia-Lin et Peter HALL (2012) «The wider spatial-economic impacts of high-speed trains : a comparative study of Manchester and Lille sub-regions», Journal of Transport Geography, vol. 24, septembre, p. 89-110.

CHEN, Zhenhua et Kingsley E. HAYNES (2012) «Tourism Industry and High Speed Rail, Is There a Linkage: Evidence from China's High Speed Rail Development ", Communication au colloque de l'ASRDLF, juillet 2012, Belfort (France), $20 \mathrm{p}$.

CORONADO, José Maria; Maddi GARMENDIA; Amparo MOYANO et José Maria URENA (2012) «Mesuring High Speed Rail Usefulness for Tourisme in Spain », Communication au colloque de l'ASRDLF, juillet 2012, Belfort (France), $18 \mathrm{p}$.

DELAPLACE, Marie et Julie PERRIN (2012) «Multiplication des dessertes TGV et tourismes urbains et d'affaires. Regards croisés sur la province et l'Île-de-France», Communication au colloque de l'ASRDLF, juillet 2012, Belfort (France), 20 p.

DIDIER, Sophie (2000) Une île dans la ville? Invention, négociation et mise en pratique du modèle de ville Disney à Anaheim (Californie), 1950-2000, Thèse de doctorat en géographie, Paris : Université Paris $1.422 \mathrm{p}$.

DIDIER, Sophie (2001) «Parcs de loisirs et nouveaux espaces publics : Le «Disneyland Resort» d'Anaheim (Californie)», dans Cynthia GHORRA-GOBIN (sous la direction de), Réinventer le sens de la ville: Les espaces publics à l'heure globale, Paris : L'Harmattan. 266 p.

DIDIER, Sophie (2002) «L'ambiguïté de l'expérience Disneyland en matière de gestion des flux de visiteurs ", Flux, nº50, p. 34-43.

DUHAMEL, Philippe et Rémy KNAFOU (2007) Mondes urbains $d u$ tourisme, Paris : Belin. 366 p.

Équipe MIT - Mobilités, itinéraires, tourisme (2002) Tourismes 1 - Lieux communs, Paris : Belin. 315 p.

Expedia (2013) «Hotels», Expedia, http://www.expedia.com/Hotels?v=a, consulté le 1 décembre 2013.

FACCHINETTI-MANNONE, Valérie et Cyprien RICHER (2011) "L'intégration territoriale des gares sur lignes à grande vitesse en France : une approche typologique», Recherche Transports Sécurité, vol. $27, \mathrm{n}^{\circ} 3$, p. 200-214.

FOUCRIER, Annick (2010) «Le mythe californien dans l'histoire américaine», Pouvoirs, n¹33, p. 5-15.

FOUCRIER, Annick et Antoine Coppolani (2004) La Californie : périphérie ou laboratoire?, Paris : L'Harmattan. 299 p.

KNAFOU, Rémy (2003) «Parc à thème», dans Jacques LÉVY et Michel LUSSAULT (sous la direction de), Dictionnaire de la géographie l'espace des sociétés, Paris : Belin. 1033 p.

LE GOIX, Renaud (2003) Les «Gated Communities» aux États-Unis. Morceaux de villes ou territoires à part entière?, Thèse de doctorat en géographie, Paris : Université Paris 1. 492 p. 
L'HOSTIS, Alain et Hervé BAPTISTE (2006) «A transport network for a City network in the Nord-Pas de-Calais region: linking the performance of the public transport service with the perspectives of a monocentric or a polycentric urban system ", European Journal of Spatial Development, $\mathrm{n}^{\circ} 20$, octobre, $18 \mathrm{p}$.

MARLING, Karal Ann (1991) «Disneyland, 1955: Just Take the Santa Ana Freeway to the American Dream ", American Art, vol. 5, n¹-2, hiver-printemps, p. 168-207.

MASSON, Sophie et Romain PETIOT (2009) «Can the high speed rail reinforce tourism attractiveness? The case of the high speed rail between Perpignan (France) and Barcelona (Spain)», Technovation, no29, p. 611-617.

METRO (2013) «Maps \& Timetable», LA Metro Home, <http://www.metro. net/riding/maps/>, consulté le 1 décembre 2013.

METROLINK (2013) «Routes», Metrolink Trains, <http://metrolinktrains. com/routes/>, consulté le 1 décembre 2013.

OCTA - ORANGE COUNTY TRANSPORTATION AUTHORITY (2012)

ARC : Anaheim Rapid Connection. Anaheim : AConnext. 1 p.

OFFNER, Jean-Marc (1993) «Les effets structurants du transport : mythe politique, mystification scientifique», L'Espace géographique, tome 22, $\mathrm{n}^{\circ} 3, \mathrm{p} .233-242$.

ORANGE COUNTY BUSINESS COUNCIL (2008) The Economic Impact of High Speed Trains for Orange County. Orange Councty Business Council. $26 \mathrm{p}$.

PERL, Anthony (2002) New Departures, Rethinking Rail Passenger Policy in the Twenty-First Century, Lexington: The University Press of Kentucky. $334 \mathrm{p}$.

PERL, Anthony (2012) «Assessing the recent reformulation of United States passenger rail policy", Journal of Transport Geography, n²2, p. 271-281.

STARR, Kevin (1985) Inventing the Dream, California through the Progressive Era, New York/Oxford: Oxford University Press. 380 p.

THEMED ENTERTAINMENT ASSOCIATION (2014) 2013 Theme Index / 2013 Museum Index, Global Attractions Attendance Report, Burbank: AECOM. 37 p.

URENA, José Maria et Cecilia RIBALAYGUA (2004) «Le réseau ferroviaire à grande vitesse espagnol : état actuel d'avancement et d'appropriation par les villes desservies», Transports Urbains, n¹06, p. 3-10.

U.S. DEPARTMENT OF COMMERCE, International Trade Administration \& Office of Travel and Tourism Industries (2012) Overseas Visitation Estimates for U.S. States, Cities, and Census Regions: 2012. Washington D.C.: Department of Commerce. 6 p.
U.S. DEPARTMENT OF TRANSPORTATION (2009) Vision for High-Speed Rail in America, High-Speed Rail Strategic Plan. Washington D.C.: Department of Transportation. 28 p.

U.S. DEPARTMENT OF TRANSPORTATION (2010) National Rail Plan, Moving Forward, A Progress Report. Washington D.C.: Department of Transportation. $27 \mathrm{p}$.

U.S. DEPARTMENT OF TRANSPORTATION (2013) FRA High-Speed Intercity Passenger Rail (HSIPR) Program, Funding Selection Summary. Washington D.C.: Department of Transportation. 8 p.

\section{Communications personnelles}

ALPINE, Michael C. et William DAVIS (2013) responsables de la construction de l'ARTIC, entretien et visite réalisés à Anaheim, le 12 avril.

BACKSTROM, Paul (2013) responsable de la politique des transports pour le $11^{\text {ème }}$ district de Los Angeles, entretien réalisé à Los Angeles, le 24 avril.

DUNN, Lucy (2012) présidente de l'Orange County Business Council, entretien réalisé à Anaheim, le 19 juin.

FOX, Stephen G. (2012) responsable du projet de grande vitesse pour la SCAG, entretien réalisé à Los Angeles, le 21 juin.

GARDNER, Mike (2013) conseiller municipal à Riverside, entretien réalisé à Riverside, le 22 avril.

GUTIERREZ, Ken (2013) conseiller municipal à Riverside, entretien réalisé à Riverside, le 18 avril.

LABRADO, Jennifer (2013), consultante chez Greengrass, responsable de la section Los Angeles-Anaheim, entretien réalisé à Santa Ana, le 28 mai.

LAI, Jamie et son équipe (2012) responsable de l'aménagement des transports et du projet ARTIC pour la municipalité d'Anaheim, entretien réalisé à Anaheim, le 28 juin.

MONTANEZ, Eugene (2013) conseiller municipal à Corona, entretien réalisé à Corona, le 28 mai.

PETREK, Jay (2013) responsable de l'aménagement des transports pour la municipalité d'Escondido, entretien réalisé à Escondido, le 29 mai.

VARTABEDIAN, Ralph (2012) journaliste au Los Angeles Times, entretien réalisé à Los Angeles, le 28 juin.

WAPNER, Alan (2013) conseiller municipal à Ontario, entretien réalisé à Ontario, le 23 mai. 